\title{
Mid-infrared Refractive Index Sensor Based on a 2D Photonic Crystal Coupled Cavity-two Waveguides
}

\author{
Hadjira Tayoub $^{1,2^{*}}$, Abdesselam Hocini $^{1}$, Ahlam Harhouz ${ }^{3}$ \\ ${ }^{1}$ Département d'Electronique, Faculté de Technologie, Université Mohamed Boudiaf de M'sila, BP.166, Route Ichebilia, \\ M'sila 28000, Algeria \\ ${ }^{2}$ Research Center in Industrial Technologies CRTI, P.O.BOX :64, Cheraga 16014, Algiers, Algeria \\ ${ }^{3}$ Université Mouloud Mammeri de TiziOuzou, Algeria
}

Corresponding Author Email: hadjira.tayoub@gmail.com

https://doi.org/10.18280/i2m.180211

Received: 9 January 2019

Accepted: 29 March 2019

\section{Keywords:}

mid-infrared, photonic crystal cavity, photonic crystal waveguide, Ri-based sensing, high sensitivity

\begin{abstract}
In this paper, a viable design of mid-infrared refractive index sensor based on photonic crystal coupled cavity-two waveguides is proposed. An increasing number of works are dedicated to investigate the behavior of refractive index sensor based on photonic crystal in the mid-infrared range. We define the sensitivity of our sensor by detecting the shift in the resonance wavelength as a function of the refractive index's variations in the region around the cavity. The purpose of this study is to design a highly sensitive mid-infrared photonic crystal sensor. Consequently, an improved sensitivity of $650 \mathrm{~nm} / \mathrm{RIU}$ (refractive index units) with a detection limit of 0.001 RIU has been obtained. The sensitivity can be improved from $394 \mathrm{~nm} / \mathrm{RIU}$ to $758 \mathrm{~nm} / \mathrm{RIU}$ with a detection limit of $0.01 \mathrm{RIU}$ in the wavelength range of $2,97 \mu \mathrm{m}$ to $3,71 \mu \mathrm{m}$ by increasing the number of the infiltrated holes. The same design has been used as a liquid sensor and a sensitivity of $550 \mathrm{~nm} / \mathrm{RIU}$ has been achieved with a detection limit of 0.001 RIU for $\mathrm{RI}=1.33$ and $\mathrm{RI}=1.331$. The properties of the sensor are simulated using the finitedifference time-domain (FDTD) method from the RSoft software package.
\end{abstract}

\section{INTRODUCTION}

Photonics crystals $(\mathrm{PhC})$ as known today have been defined for the first time by E.Yablonovitch [1] and S. John [2], they have inspired inclusive interest for as far back as decades because of the existence of photonic bandgap (PBG) and the ability to control the propagation of the electromagnetic (EM) waves inside it; which is extremely required for designing PCbased optical devices [3]. $\mathrm{PBG}$ in $\mathrm{PhC}$ prohibits the propagation of light in the material. Frequencies falling in the bandgap are not permitted to propagate because of the inexistence of the optical modes. Yet, introducing certain defects such as point defects as cavities or line defects as waveguides, and their coupled elements in the structure can adjust the dispersion diagram hence permit to particular modes to propagate in the bandgap [4].

The interaction between a cavity resonator and a waveguide system has been previously demonstrated in [5-8], tremendous research works are done in biosensing [9-14], chemical and gas sensing [15-17], temperature sensing [18], stress sensing [19]. It was also shown that a $\mathrm{PhC}$ cavity and waveguides sensor might be used in refractive index (RI) sensing where the detection of an analyte may be done by a local RI shift [2021].

In the few past years, photonic crystal structure in the midinfrared (mid-IR) spectral range has gained worldwide attention of scientific community due to the transparency window of silicon for the mid-IR wavelength range (between 1.1 and $8.5 \mu \mathrm{m}$ ) as proposed by Soref [22]. On the other hand, the mid-IR range is highly interesting for chemical and gas sensing, since photon energies here match well with molecular vibrations [23, 24]. However, mid-IR sensors based on photonic crystal are comparatively unexplored when compared to the near-IR and visible sensors, it is therefore of great necessity to provide a mid-IR RI sensor based on PhC coupled cavity-waveguide with high sensitivity.

Herein, a novel mid-IR refractive index sensor based on $\mathrm{PhC}$ coupled cavity-two waveguides is designed and described. Sensing technique is established on the change of the refractive index of holes in the range of $n=1.01$ to $n=1.04$, and as a result, the detection of the shifted resonance wavelength $\lambda_{0}$. The comportment of the designed sensor is analyzed using the finite-difference time-domain (FDTD) method from the RSoft software package.

\section{SENSOR DESIGN}

To investigate mid-IR refractive index sensing, a sketch of a two-dimensional RI sensor based on $\mathrm{PhC}$ coupled cavity-two waveguides composed by using a hexagonal lattice structure has been proposed in figure 1 . In the proposed structure, the size of photonic crystal is $(39 \times 21)$, the lattice constant (a) equal to $1 \mu \mathrm{m}$, the effective index profile of the silicon slab is $\mathrm{n}_{\mathrm{si}}=2.654$, the ration of the radii of air holes ( $\mathrm{r}$ ) and lattice constant is 0.3 , where the radius of the defected holes is $\mathrm{r}_{1}=0,45 \mathrm{a}$. The studied sensor is made up of two waveguides couplers and a cavity; the basic two waveguides are a $\Gamma-\mathrm{K}$ directional waveguides formed by removing one row of air holes. 


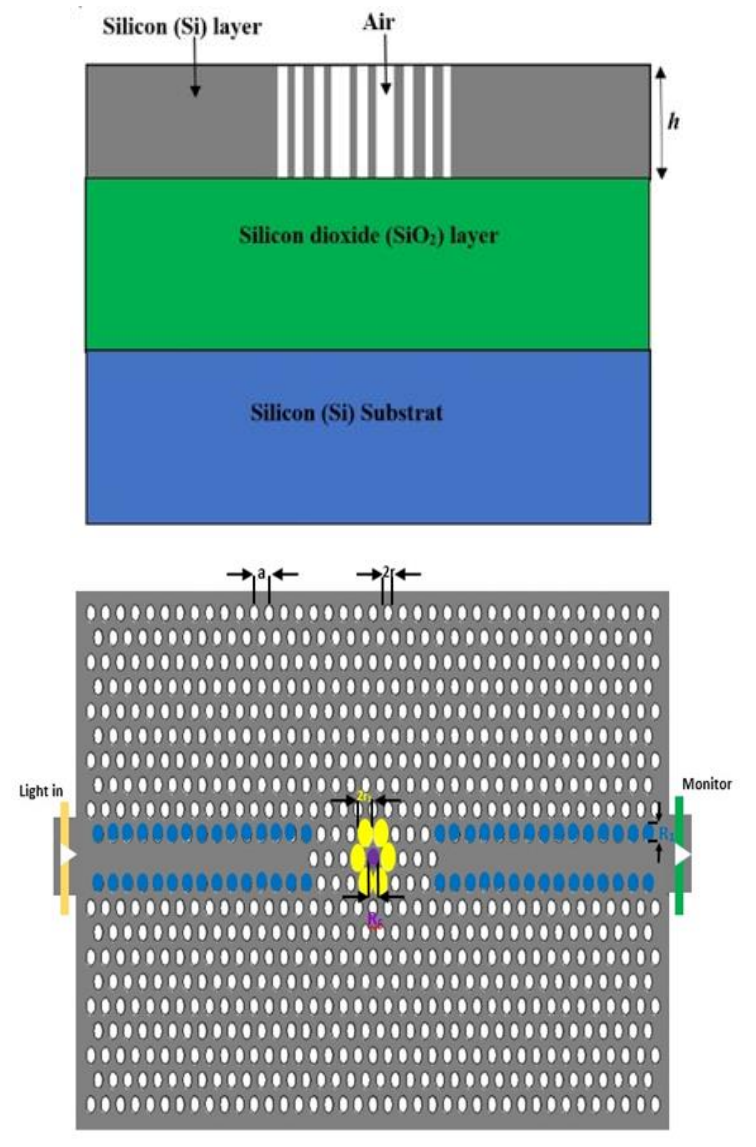

Figure 1. Designed photonic crystal coupled cavity-two waveguides sensor

\section{NUMERICAL RESULTS AND DISCUSSION}

As it was proved by Edwards et al. [25], by using the effective index method the 2D analysis would be close similar to the 3D analysis. The effective index of the proposed structure has been taken equal to 2,654 for a silicon guiding layer with a central wavelength of $3600 \mathrm{~nm}$.

The designed photonic crystal structure having band gap in the wavelength range of $2,9736 \mu \mathrm{m}$ to $3,7145 \mu \mathrm{m}$ for TEpolarized wave; which is calculated by 2D Plane Wave Expansion (PWE) method. The photonic band structure principally depends on the radius of holes and the lattice constant of the structure.

Several approaches are used to create a cavity resonance such as increasing or reducing the radius of particular holes [26]. In the first design A, a microcavity was created within the structure by changing the radius of the holes surrounding the central hole (showing in yellow color in figure 1) to confine light within the cavity, and by separating the microcavity by three holes of the both sides of the waveguides.

In this sensor, we used the input of the waveguide to apply a Gaussian pulse around 3,6 $\mu \mathrm{m}$ to excite the resonant modes of the cavity. In addition, a power monitor was positioned at the end of the waveguide to monitor the trapped mode in order to determine the sensitivity of the proposed sensor [27].

Based on the designed device, the transmittance spectra corresponding the refractive index $n=1$ is represented in figure 2.a. The result presents two different resonant peak wavelengths at $3,39 \mu \mathrm{m}$ and $3,6 \mu \mathrm{m}$, A resonant wavelength of $3,39 \mu \mathrm{m}$ is selected since it has the highest sensitivity. Figure 2.b illustrate the $\mathrm{y}$-component electric field (Ey) distribution of PC cavity in $\mathrm{x}-\mathrm{y}$ plane. As seen, the TE-like polarized light is strongly confined in the $\mathrm{x}$-direction.

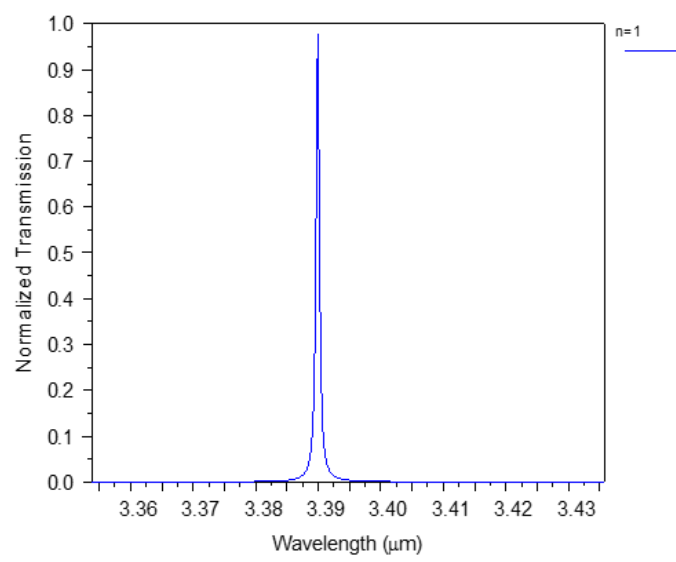

(a)

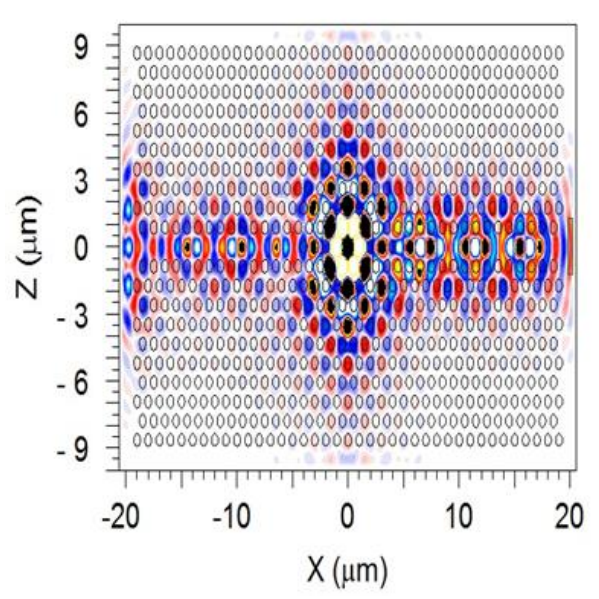

1.0

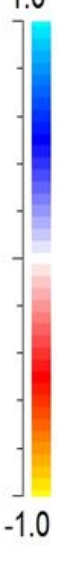

(b)

Figure 2. (a) Transmittance of the designed device for $n=1$. (b) The y-component electric field (Ey) distribution of PC cavity in $x-y$ plane

The result of the FDTD simulation with a grid size of 0.05 of the proposed structure is shown in figure 3 . The normalized transmission spectrum of the resonant mode peak wavelengths for different refractive indices shows a shift in the resonance wavelength of $6.5 \mathrm{~nm}$ for $\Delta \mathrm{n}=0.001$ and a sensitivity of 650 $(\mathrm{nm} / \mathrm{RIU})$ is observed (Table 1$)$.

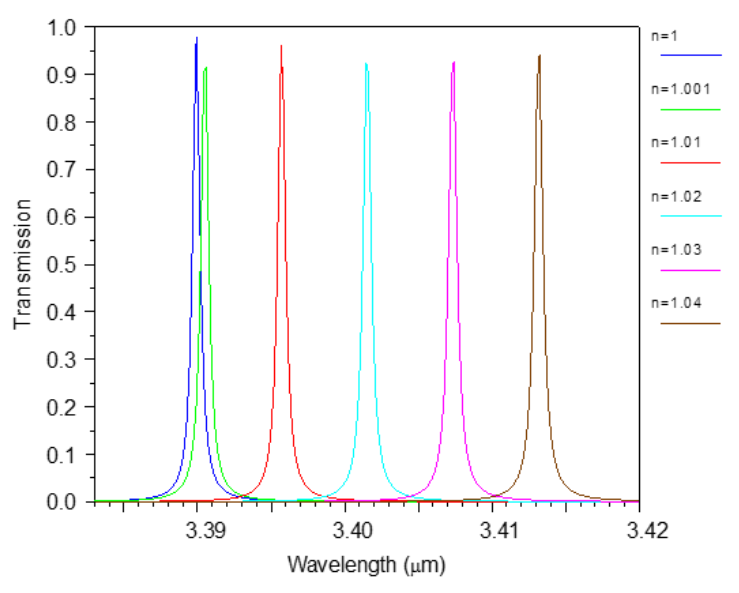

Figure 3. Normalized transmission spectrum of the proposed design as a function of wavelength for different refractive indices 
Table 1. The sensitivity of the sensor for different RI

\begin{tabular}{ccc}
\hline Refractive Index & $\begin{array}{c}\text { Sensitivity } \\
(\mathbf{n m} / \mathbf{R I U})\end{array}$ & $\Delta \mathbf{n}(\mathbf{R I U})$ \\
\hline 1 & - & $\mathrm{n} 0$ \\
1.001 & 650 & 0.001 \\
1.01 & 575 & 0.01 \\
1.02 & 576.5 & 0.02 \\
1.03 & 583 & 0.03 \\
1.04 & 582.5 & 0.04 \\
1.33 & 550 & 0.001 \\
\hline
\end{tabular}

A microcavity is created in the structure by increasing the central hole's radius $\left(\mathrm{R}_{\mathrm{C}}\right)$ (shown in magenta color in figure.1) for $\mathrm{R}_{\mathrm{C}}$ equal to $0.27,0.28$, and $0.29 \mu \mathrm{m}$.

The simulation results reveal that sensitivities of 577,592 and $592(\mathrm{~nm} / \mathrm{RIU})$ are achieved at $\mathrm{R}_{\mathrm{C}}$ equal to $0.27,0.28 \mu \mathrm{m}$ and $0.29 \mu \mathrm{m}$, respectively (figure 4 ). By increasing the radius of the central hole, an acceptor defect state pulled from the dielectric band into the band-gap, is excited [28].

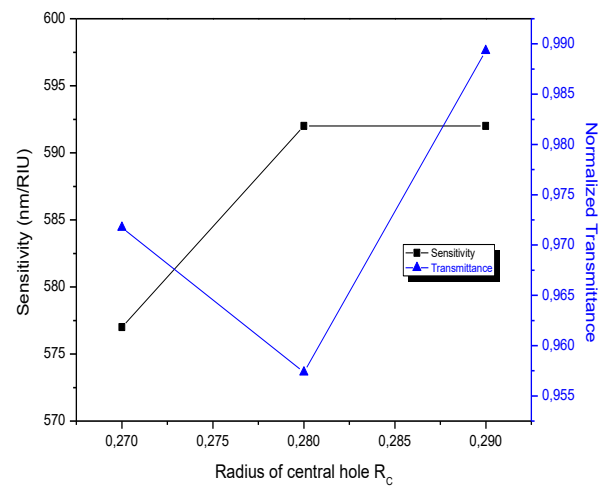

Figure 4. Relationships of the sensitivity and the normalized transmittance of our sensor to radius of central hole $\mathrm{R}_{\mathrm{C}}$

In order to achieve a better sensitivity, the mentioned above design $\mathrm{A}$ was changed to the design $\mathrm{B}$. In design $\mathrm{B}$, the radii of the holes positioned in the vicinity of the two-waveguides (shown in blue color in figure 1) can therefore be enlarged to benefit from an improved light-matter interaction level [18]. As seen in figure 5, the simulation results reveal that a sensitivity of $623(\mathrm{~nm} / \mathrm{RIU})$ is achieved at $\mathrm{R}_{1}$ equal to $0.35 \mu \mathrm{m}$.

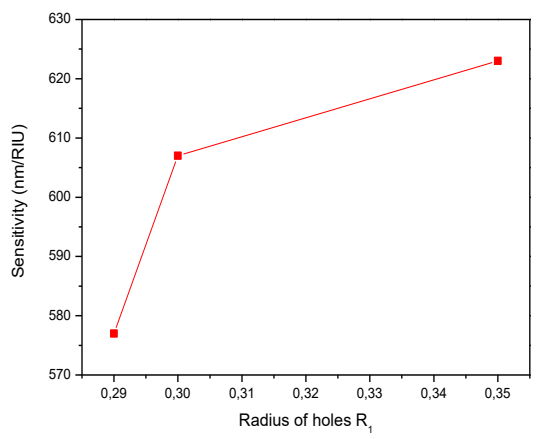

Figure 5. Variation of sensitivity as a function of the different radiuses of the first row of holes $\mathrm{R} 1$

As mentioned, previous, our main goal is to design a new mid-IR refractive index sensor based on $\mathrm{PhC}$ coupled cavitytwo waveguides with the highest possible sensitivity. In this section, as seen in figure 6.a, the sensing properties of the studied sensor were investigated by changing the number of functionalized holes $\mathrm{N}(6,12,18$ and 28$)$ when $\mathrm{r}_{1}=0,45 \mathrm{a}$.

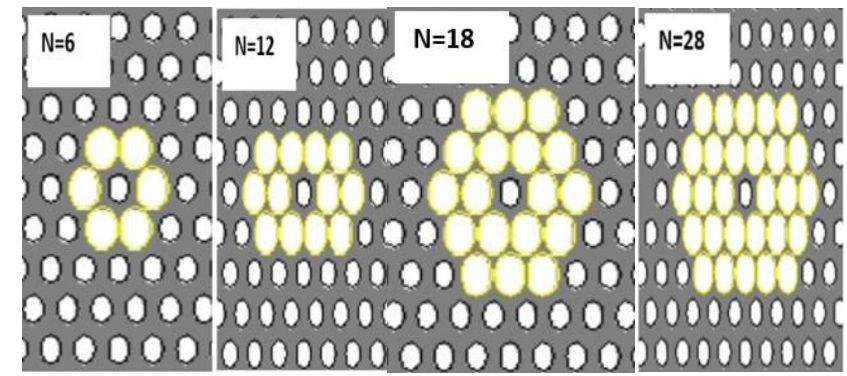

(a)

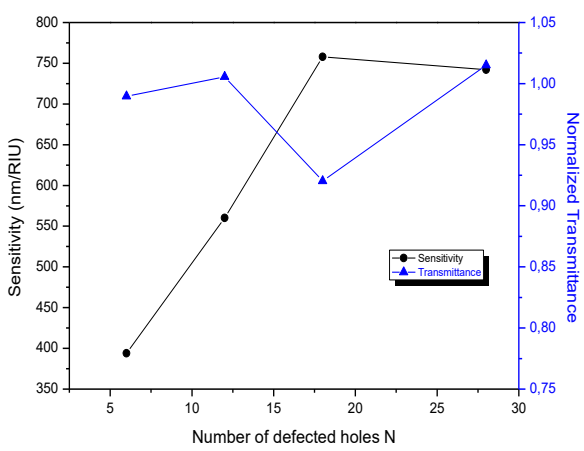

(b)

Figure 6. (a) Schematic diagrams of defected holes when $\mathrm{N}=6,12,18$ and 28 (holes in yellow color refers to the sensing region filled with analyte), (b) Relationships of the sensitivity and the normalized transmittance of our improved sensor to number of defected holes $\mathrm{N}$

Table 2. Comparison of the proposed sensor with several similar $\mathrm{PhC}$ designs

\begin{tabular}{cccc}
\hline Sensing structure & $\begin{array}{c}\text { Sensitivity } \\
(\mathbf{n m} / \mathbf{R I U})\end{array}$ & $\begin{array}{c}\text { detection } \\
\text { limit } \\
\text { (RIU) }\end{array}$ & Year \\
\hline $\begin{array}{c}\text { [30] RI biosensor formed } \\
\text { by two waveguides and } \\
\text { one microcavity. }\end{array}$ & 330 & 0.001 & 2008 \\
\hline $\begin{array}{c}\text { [31] RI sensor formed by } \\
\text { Two types of defect } \\
\text { nanocavities L3 and H1-r } \\
\text { embedded between two } \\
\text { W1 photonic crystal } \\
\text { waveguides }\end{array}$ & $70-200$ & 0.018 & 2008 \\
\hline $\begin{array}{c}\text { [17] RI sensor formed by } \\
\text { PhC microcavity }\end{array}$ & 500 & 0.05 & 2016 \\
\hline $\begin{array}{c}\text { [32] PhCW based RI } \\
\text { sensor }\end{array}$ & 260 & 0.001 & 2013 \\
\hline $\begin{array}{c}\text { [13] RI biosensor formed } \\
\text { by two waveguides and } \\
\text { one microcavity }\end{array}$ & 425 & 0.001 & 2015 \\
\hline The proposed sensor & 650 & 0.001 & - \\
\hline
\end{tabular}

As it can be expected, a sensitivity of $758 \mathrm{~nm} / \mathrm{RIU}$ has been achieved, the sensitivity increases rapidly to a greater number of functionalized holes (figure 6.b). this can be explained by the reduced effective refractive index between the infiltrated holes and the semiconductor membrane [14, 29]. Consequently, a local infiltration guarantees a better sensitivity. 
A brief comparison between our results and some of the results already published in scientific research papers is illustrated in Table 2.

These results display that the proposed sensor possesses a better sensing property and well apply to RI sensing in mid-IR

\section{CONCLUSIONS}

To conclude, we have effectively designed and simulated in this paper a novel mid-IR 2D photonic crystal coupled cavitytwo waveguides platform for RI-based sensing application nearby $\lambda_{0}=3.6 \mu \mathrm{m}$ and the extensive simulation results show that the proposed sensor is highly sensitive to a small change in refractive index, a high sensitivity of $758 \mathrm{~nm} / \mathrm{RIU}$ in the RI range of 1.01-1.04 was accomplished. Due to the improvement in the limit of detection with high sensitivity, the high transmittance and the small size, our improved sensor is well matched for applications in mid-IR sensing and it would be a great potential platform in other on-chip photonic circuits. Improving the achieved sensitivity by using slotted waveguide coupled cavity sensor will be subject of future studies.

\section{REFERENCES}

[1] Yablonovitch, E. (1987). Inhibited spontaneous emission in solid-state physics and electronics. Physical Review Letters, 58(20): 2059-2062 https://doi.org/10.1103/PhysRevLett.58.2059

[2] John, S. (1987). Strong localization of photons in certain disordered dielectric superlattices. Physical Review Letters, 58(23): 2486-2489. https://doi.org/10.1103/PhysRevLett.58.2486

[3] Chhipa, M.K, Radhouene, M., Robinson, S., Suthar, B. (2017). Improved dropping efficiency in twodimensional photonic crystal-based channel drop filter for coarse wavelength division multiplexing application. Optical Engineering, 56(1): 015107. https://doi.org/10.1117/1.OE.56.1.015107

[4] Goyal, A.K., Duttaa, H.S., Pal, S. (2017). Recent advances and progress in photonic crystal based gas sensors. Journal of Physics D: Applied Physics, 50(20). https://doi.org/10.1088/1361-6463/aa68d3

[5] Olivier, S.N., Smith, C.J.M., Benisty, H., Weisbuch, C., Krauss, T.F., Houdre, R., Oesterle, U. (2002). Cascaded photonic crystal guides and cavities: Spectral studies and their impact on integrated optics design. IEEE Journal of Quantum Electronics, 38(7): 816-824. https://doi.org/10.1109/JQE.2002.1017592

[6] Kim, G., Lee, Y. (2004). Coupling of small, low-loss hexapole mode with photonic crystal slab waveguide mode. Optics Express, 12(26): 6624-6631. https://doi.org/10.1364/OPEX.12.006624

[7] Okano, M., Kako, S., Noda, S. (2003). Coupling between a point-defect cavity and a line-defect waveguide in three-dimensional photonic crystal. Physical Review B, 68(23):

$1-10$. https://doi.org/10.1103/PhysRevB.68.235110

[8] Zhang, Z., Qiu, M. (2005). Compact in-plane channel drop filter design using a single cavity with two degenerate modes in 2D photonic crystal slabs. Optics Express, 13(7): 2596-2604 https://doi.org/10.1364/OPEX.13.002596
[9] Skivesen, N., Horvath, R., Thinggaard, S., Larsen, N.B., Pedersen, H.C. (2007). Deep-probe metal-clad waveguide biosensors. Biosensors and Bioelectronics, 22(7): 1282-1288. https://doi.org/10.1016/j.bios.2006.05.025

[10] Lee, P.T., Lu, T.W., Yu, C.M., Tseng, C.C. (2007). Photonic crystal circular-shaped microcavity and its uniform cavity-waveguide coupling property due to presence of whispering gallery mode. Optics Express, 15(15): 9450-9457. https://doi.org/10.1364/OE.15.009450

[11] Kurt, H., Yilmaz, D.Y., Akosman, A.E., Ozbay, E. (2012). Asymmetric light propagation in chirped photonic crystal waveguides. Optics Express, 20(18): 20635. https://doi.org/10.1364/OE.20.020635

[12] Areed, N.F.F., Hameed, M.F.O., Obayya, S.S.A. (2017). Highly sensitive face-shaped label-free photonic crystal refractometer for glucose concentration monitoring. Optical and Quantum Electronics, 49(1): 1-12. https://doi.org/10.1007/s11082-016-0847-9

[13] Harhouz, A., Hocini, A. (2015). Design of high-sensitive biosensor based on cavity-waveguides coupling in 2D photonic crystal. Journal of Electromagnetic Waves and Applications, 29(5): 659-667. https://doi.org/10.1080/09205071.2015.1012597

[14] Arafa, S., Bouchemat, M., Bouchemat, T., Benmerkhi, A., Hocini, A. (2017). Infiltrated photonic crystal cavity as a highly sensitive platform for glucose concentration detection. Optics Communications, 384: 93-100. https://doi.org/10.1016/j.optcom.2016.10.019

[15] Yang, D., Tian, H., Ji, Y. (2011). Nanoscale photonic crystal sensor arrays on monolithic substrates using sidecoupled resonant cavity arrays. Optics Express, 19(21): 11709-11717. https://doi.org/10.1364/OE.19.020023

[16] Zhang, Y., Zhao, Y., Lv, R. (2015). A review for optical sensors based on photonic crystal cavities. Sensors Actuators A: Physical, 233: 374-389. https://doi.org/10.1016/j.sna.2015.07.025

[17] Goyal, A.K., Dutta, H.S., Pal, S. (2016). Design and analysis of photonic crystal micro-cavity based optical sensor platform. AIP Conference Proceedings, 1724(1): 020005. https://doi.org/10.1063/1.4945125

[18] Hocini, A., Harhouz, A. (2016). Modeling and analysis of the temperature sensitivity in two-dimensional photonic crystal microcavity. Journal of Nanophotonics, 10(1): 016007. https://doi.org/10.1117/1.JNP.10.016007

[19] Yang, Y., Yang, D., Tian, H., Ji, Y. (2013). Photonic crystal stress sensor with high sensitivity in double directions based on shoulder-coupled aslant nanocavity. Sensors Actuators: A Physical, 193: 149-154. https://doi.org/10.1016/j.sna.2013.01.045

[20] Buswell, S.C., Wright, V.A., Buriak, J.M., Van, V., Evoy, S. (2008). Specific detection of proteins using photonic crystal waveguides. Optics Express, 16(20): 1594915957. https://doi.org/10.1364/OE.16.015949

[21] Zhao, Y., Zhang, Y., Wu, D., Wang, Q. (2012). Fiber loop ring-down refractive index sensor based on high-Q photonic crystal cavity. IEEE Sensors Journal, 14(6): 36. https://doi.org/10.1109/JSEN.2014.2305971

[22] Soref, R. (2010). Mid-infrared photonics in silicon and germanium Nat. Photonics, 4(8): 495-497. https://doi.org/10.1038/nphoton.2010.171

[23] Liu, F.M., Lu, W., Cai, Z.Y. (2018). Design and implementation of an infrared radiation sensor based on 
STC12C5A. Instrumentation, Mesure, Metrologie, 17(4): 593-603. https://doi.org/10.3166/I2M.17.593-603

[24] Blanco-Redondo, A., Sarriugate, P., Garcia-Adeva, A., Zubia, J., Hillenbrand, R. (2014). Coupling mid-infrared light from a phCotonic crystal waveguide to metallic transmission lines. Applied Physics Letters, 104(1): https://doi.org/10.1063/1.4859635

[25] Edwards, D.F., Ochoa, E. (1980). Infrared refractive index of silicon. Applied Optics, 19(24): 4130-4131. https://doi.org/10.1364/AO.19.004130

[26] Pal, S., Guillermain, E., Sriram, R., Miller, B., Fauchet, P.M. (2010). Microcavities in photonic crystal waveguides for biosensor applications. Frontiers in Pathogen Detection: From Nanosensors to Systems, 7553: 755304-10. https://doi.org/10.1117/12.848237

[27] Akahane, Y., Asano, T., Song, B., Noda, S. (2003). High$\mathrm{Q}$ photonic nanocavity in a two-dimensional photonic crystal. Nature, 425: 944-947. https://doi.org/10.1038/nature02015.1

[28] Yablonovitch, E., Gmitter, T.J., Meade, R.D., Rappe, A.M., Brommer, K.D., Joanopoulos, J.D. (1991). Donor and acceptor modes in photonic band structure. Physical Review Letters, 67(24): 3380-3383
https://doi.org/10.1103/PhysRevLett.67.3380

[29] Zhang, Y., Zhao, Y., Wang, Q. (2015). Measurement of methane concentration with cryptophane $\mathrm{E}$ infiltrated photonic crystal microcavity. Sensors Actuators B: Chemical, 209: 431-437. https://doi.org/10.1016/j.snb.2014.12.002

[30] Wang, X., Xu, Z., Lu, N., Zhu, J., Jin, G. (2008). Ultracompact refractive index sensor based on microcavity in the sandwiched photonic crystal waveguid. Optics Communications, 281(6): 1725-1731. https://doi.org/10.1016/j.optcom.2007.11.040

[31] Dorfner, D.F., Hurlimann, T., Zabel, T., Frandsen, L.H., Abstreiter, G., Finley, J.J. (2008). Silicon photonic crystal nanostructures for refractive index sensing. Applied Physics Letters, 93(18): 2006-2009. https://doi.org/10.1063/1.3009203

[32] Dutta, H.S., Pal, S. (2013). Design of a highly sensitive photonic crystal waveguide platform for refractive index based biosensing. Optical and Quantum Electronics, 45(9): 907-917. https://doi.org/10.1007/s11082-0139697-x 